\title{
Compact Fluorescence Detection System for Polymerase Chain Reaction Chips
}

\author{
Deuk-Ju Lee, ${ }^{1,2}$ Ji-Soo Hwang, ${ }^{1,2}$ Ji-Seong Park, ${ }^{1,2}$ Chan-Young Park, ${ }^{1,2}$ \\ Hye-Jeong Song, ${ }^{1,2}$ Yu-Seop Kim, ${ }^{1,2}$ and Jong-Dae Kim ${ }^{1,2^{*}}$ \\ ${ }^{1}$ School of Convergence Software, Hallym University, 1, Hallimdaehak-gil, \\ Chuncheon-si, Gangwon-do 24252, Korea \\ ${ }^{2}$ Bio-IT Research Center, Hallym University, 1, Hallimdaehak-gil, \\ Chuncheon-si, Gangwon-do 24252, Korea \\ (Received April 16, 2018; accepted March 20, 2019)
}

Keywords: DNA detection, PCR chip, fluorescence, optic, excitation, emission

In this paper, we propose a compact system for the fluorescence detection of polymerase chain reaction (PCR) chips. Various fluorescence detection methods have been proposed, but all require many optical components and optical distances. In contrast, in this paper, we propose a system for detecting fluorescence by attaching an LED and a photodiode directly to the reagent surface. The system is very compact and inexpensive because it requires only excitation and emission filters. To optimize the performance of the proposed system, LED and photodiode arrangements, selection of filters, and signal amplification circuits were investigated. The modification of the PCR protocol to obtain better results was also investigated. PCR amplification experiments on real DNA samples showed that the proposed system works well.

\section{Introduction}

Polymerase chain reaction (PCR) is an important technique for biological research because it can be used to test for diseases with a small amount of DNA. ${ }^{(1-4)}$ It is used in a wide range of fields such as the diagnosis of infectious diseases caused by bacteria, viruses, and fungi, and in criminal investigations. The DNA detection process requires four steps: DNA extraction, DNA amplification, electrophoresis, and gel image analysis. Generally, PCR amplifies DNA and analyzes the results through several processes. ${ }^{(5-7)}$

However, this process is time-consuming and the sample is likely to be contaminated through several manual DNA detection steps. Real-time PCR was developed to overcome these drawbacks. The amount of nucleic acid amplification products can be detected in real time and the results can be analyzed in a time shorter than that by conventional methods. ${ }^{(8,9)}$ The possibility of contamination can also be minimized since no manual steps such as opening the reaction tube to move the reagent to the detection device are required. Therefore, there are many studies on real-time PCR and various devices are being sold. ${ }^{(10)}$

*Corresponding author: e-mail: kimjd@hallym.ac.kr

http://dx.doi.org/10.18494/SAM.2019.2284 
The fluorescence detection device in the gel image analysis of DNA includes a method of using various sensors other than the photodiode and an image detection method based on a camera. ${ }^{(11,12)}$ Camera-based devices are expensive and large, making it difficult to conduct experiments. ${ }^{(13-15)}$ The real-time gene amplification test equipment used in hospitals and life sciences is generally large. Therefore, various fluorescence detection methods based on photodiodes are being tried, and many studies are under way.

Lab-on-a-chip (LOC) is being developed to make biochemical processes inexpensive and compact. $^{(16-18)}$ LOC is known as a technology that can handle small volumes of samples, meaning "I put the lab on one chip". Therefore, the production cost should be reduced and a small amount of sample processing must be possible. Because of this demand, the development of microfluidic channels has become more active. Therefore, channels using various materials have been developed, and more experiments using flexible materials and tapes that are easy to manufacture have been carried out. ${ }^{(19,20)}$ Since tapes are already available in many colors and thicknesses, they are easier to manufacture than PDMS or PMMA. ${ }^{(21,22)}$

Various fluorescence detection methods for PCR chips have been reported. Typical examples are dichroic-mirror-based illumination and side-illumination methods for miniaturization. ${ }^{(23)}$ There are various kinds of sensors for fluorescence detection. Generally, there are methods using a photodiode and a lens, and a camera including a CCD or a CMOS sensor. ${ }^{(24)}$

Various real-time PCR systems have been developed for the purpose of miniaturization. However, most fluorescence detection methods require many optical components and distances for image detection. To solve this problem, we propose a micro real-time PCR system that detects fluorescence by directly attaching an LED and a photodiode to a PCR chip containing a DNA sample. This system minimizes the number of optical components required in conventional fluorescence detection systems and is constructed using only excitation and emission filters. We also looked at the optimal positions of the LED and photodiode, and examined other filters with a similar performance to the existing expensive interference filters. The optical signal amplification circuit and protocol modification of PCR are also discussed to solve problems caused by the simple optical structure of the proposed system. In addition, as DNA amplification requires very precise temperature control to ensure reliable, reproducible, and sensitive PCR results, a compensation method is investigated to overcome the temperature error between the temperature of the reagent and the thermistor sensor outside the chamber.

Although the proposed system could be implemented in a smaller size and a lower price than the conventional real-time PCR chip, experimental results showed that the actual DNA amplification performed well.

\section{Materials and Methods}

\subsection{PCR chip and temperature compensation of fluidic channel}

The PCR chip employed in this paper has a 6-layer structure consisting of a printed circuit board (PCB), a packing tape, a double-sided tape, a plastic cover, a plastic housing, and a transparent double-sided tape connecting the housing and PCB (Fig. 1). The tapes and films 
were selected after sufficient experimentation for PCR inhibition and efficiency. Figure 1 shows the structure of the PCR chip. A $200 \mu \mathrm{m}$ PCB with a heater pattern and a thermistor is bonded on the housing, which serves to block the reagent injection inlet and light, using the double-sided tape. A fluidic channel was constructed using a $5 \mu \mathrm{m}$ boxing tape (\# 309, 3M, USA) and a $400 \mu \mathrm{m}$ double-sided tape (\# 5629BWN, Nitto, Japan). The cover film was made of polypropylene of $500 \mu \mathrm{m}$ thickness.

The NTC thermistor is attached to the bottom of the PCB substrate to measure the temperature of the fluidic channel during DNA amplification. Therefore, there is a temperature difference between the reagent and thermistor in the fluidic channel. Therefore, in this study, a commercially available thermocouple was used to confirm the reagent temperature (Fig. 2). The temperature of the reagent was measured using a thermocouple reader (TC-08, Omega Co., Ltd., USA) and a thin, 0.050-mm-diameter, K-type thermocouple (CHAL-002, Omega Co., Ltd., USA) without coating. The thermocouple reader had a conversion time of $100 \mathrm{~ms}$ and a temperature accuracy of $\pm 0.5^{\circ} \mathrm{C}$. The thermocouple was placed at the center between the box tape and the double-sided tape as shown in Fig. 3.

We implemented the program directly using the library provided by the reader manufacturer when measuring the internal temperature. It was also necessary to measure two temperatures simultaneously to measure the reagent temperature inside the PCR chip chamber and compare it with the NTC thermistor temperature. Reliability is important when comparing the temperatures of both sensors.
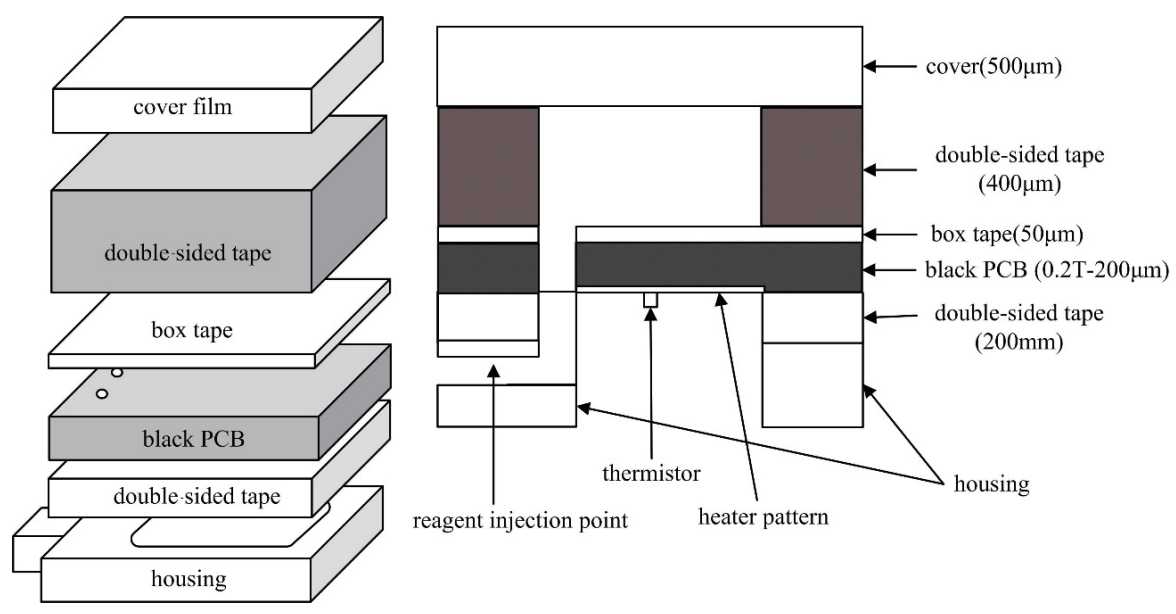

Fig. 1. (Color online) Structure of PCR chip.

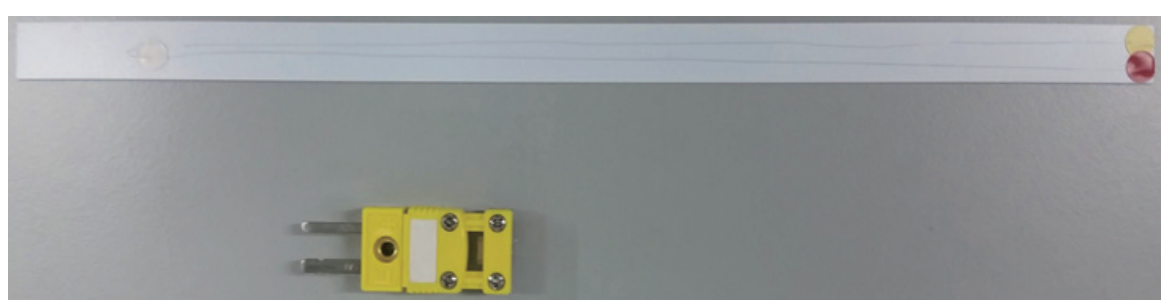

Fig. 2. (Color online) Bare-wire-type thermocouple (top) and thermocouple connector (bottom). 


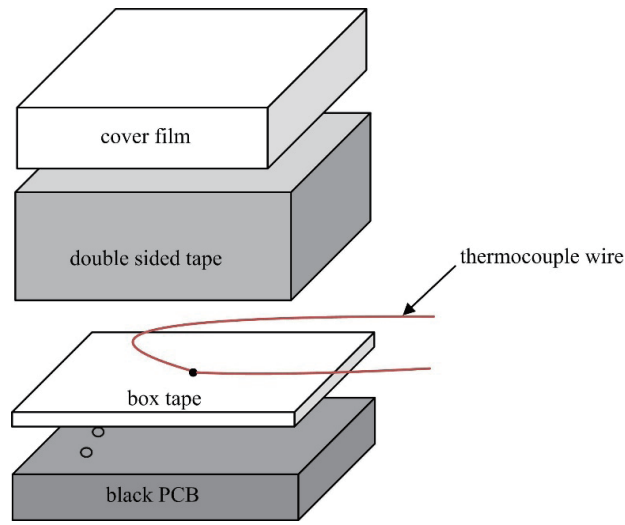

Fig. 3. (Color online) Thermocouple wire insertion diagram.

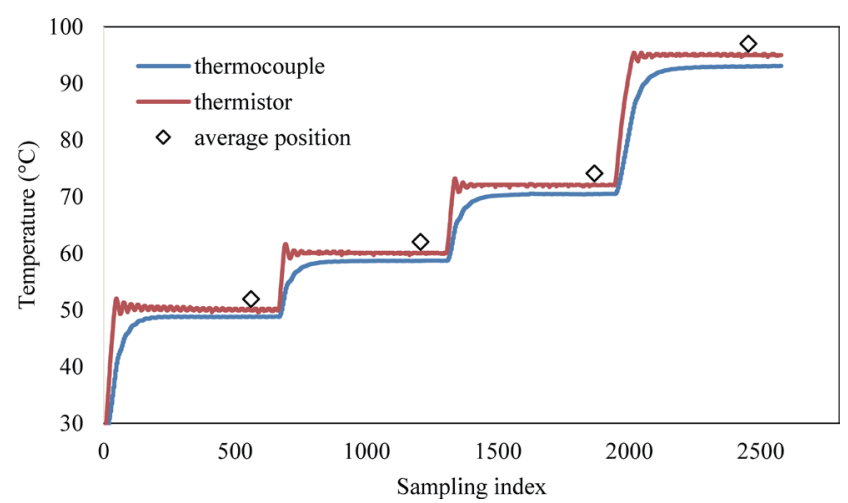

Fig. 4. (Color online) NTC thermistor and thermocouple temperature profiles.

The internal temperature was measured using a simplified protocol instead of the PCR protocol for actual DNA amplification, as only the temperature control ability can be verified. The temperature was maintained for $60 \mathrm{~s}$ at the important PCR temperatures, namely, 50, 60, 72, and $95^{\circ} \mathrm{C}$, as deduced from Fig. 4 .

In Fig. 4, the thermocouple and thermistor temperatures are denoted by the red and blue curves, respectively. The average temperature of 20 samples at the end (around the area marked ' $\checkmark$ ') of each control temperature was used to compare the two temperature differences.

\subsection{Optic module}

Figure 5 shows the integrated structure of the PCR chip and the optic module. The optic module used an optic PCB, an acrylic and silicon structure that served as a darkroom, and a 1990 mod LED (XZFBB78W, SunLED Co., Ltd., Hong Kong) that illuminated the reagents contained in the chamber. The photodiode (ODD-900-002, Opto Diode Co., Ltd., USA) was for measuring fluorescence through the emission filter (Wratten No.12, Kodak Co., Ltd., USA), and the excitation filter (ET470/40x Chroma Co., Ltd., USA) was used to filter blue light. The excitation filter is mainly used to block the wavelength of blue light passing through the emission filter. The excitation filter had a size of $2 \times 2 \times 1 \mathrm{~mm}^{3}$.

Figure 6 shows the relationship between the 3D model of the integrated module and the actual picture of each layer. Each of the above model figures shows the remaining part when removing the layer or layer group shown in the actual picture below. For example, when the housing corresponding to the actual picture displayed below the leftmost 3D model is removed, it is converted to the right $3 \mathrm{D}$ model. From this figure, the components, such as the housing, PCR chip, and optic module, can be identified in detail. A black matt double-sided tape (C-1 TYPE, Jinheung Trade, South Korea) was used for the reservoir depicted at the center of the 'channel' group. A paper tape (MATTE TYPE, Jinheung Trade, South Korea) was used for adhesion between the silicone and the acrylic of the 'dark box' group. Note that it was important that the layers adhered to each other well not to leak light or be ripped off by heat. 


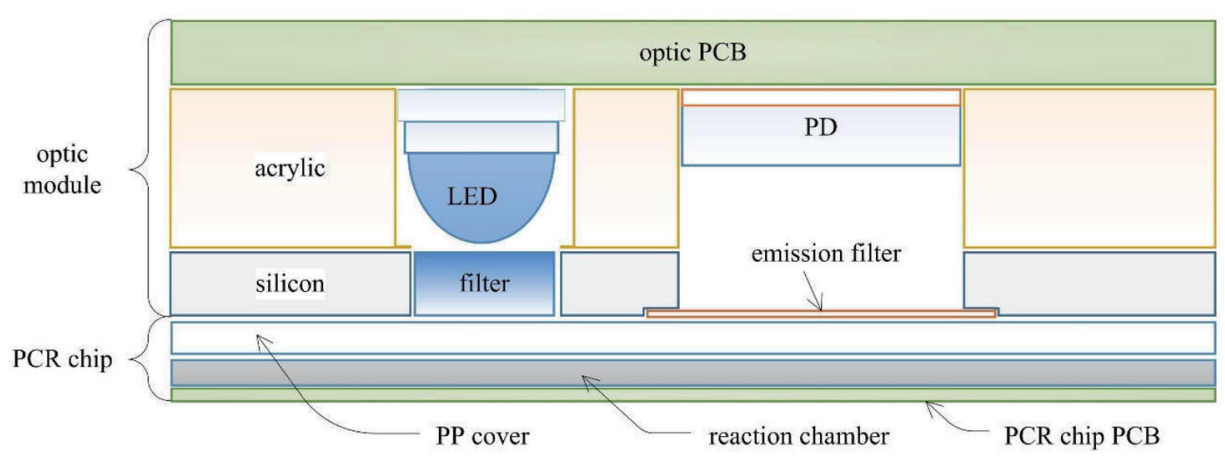

Fig. 5. (Color online) Structure of integrated module.

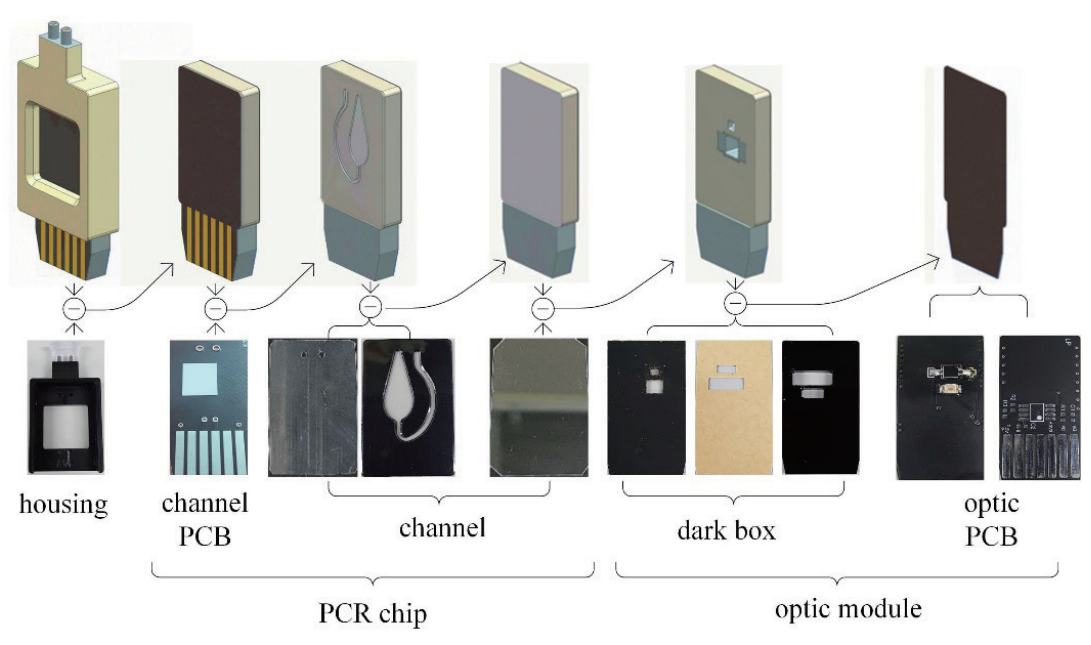

Fig. 6. (Color online) 3D model of the integrated module.

\subsection{Real-time PCR system}

Figure 7 shows the real-time PCR system including the driving system of the integrated module proposed in this paper. The local system for the real-time PCR system consists of only one microcontroller (PIC18F4553, Microchip, Taiwan) besides simple drivers or an amplifier, and the user interface can be provided by a standard computing device connected by a standard link. Figure 8 shows the layout of the fan and the integrated module. In this study, the local system was connected via a USB to a PC with a Microsoft Windows operating system. The fluidic channel of the PCR chip adopted in this study was constructed on the PCB with a heater pattern and a thermistor as described above. To drive the temperature profile, the heating or cooling of the reagent must be controlled. To obtain the resistance of an NTC thermistor mounted on a PCB board, the thermistor was connected in series with a given precision resistor, and the reference voltage of the analog-to-digital converter (ADC) was applied to measure the voltage ratio. The measured voltage division ratio was sent to the host system to obtain the 


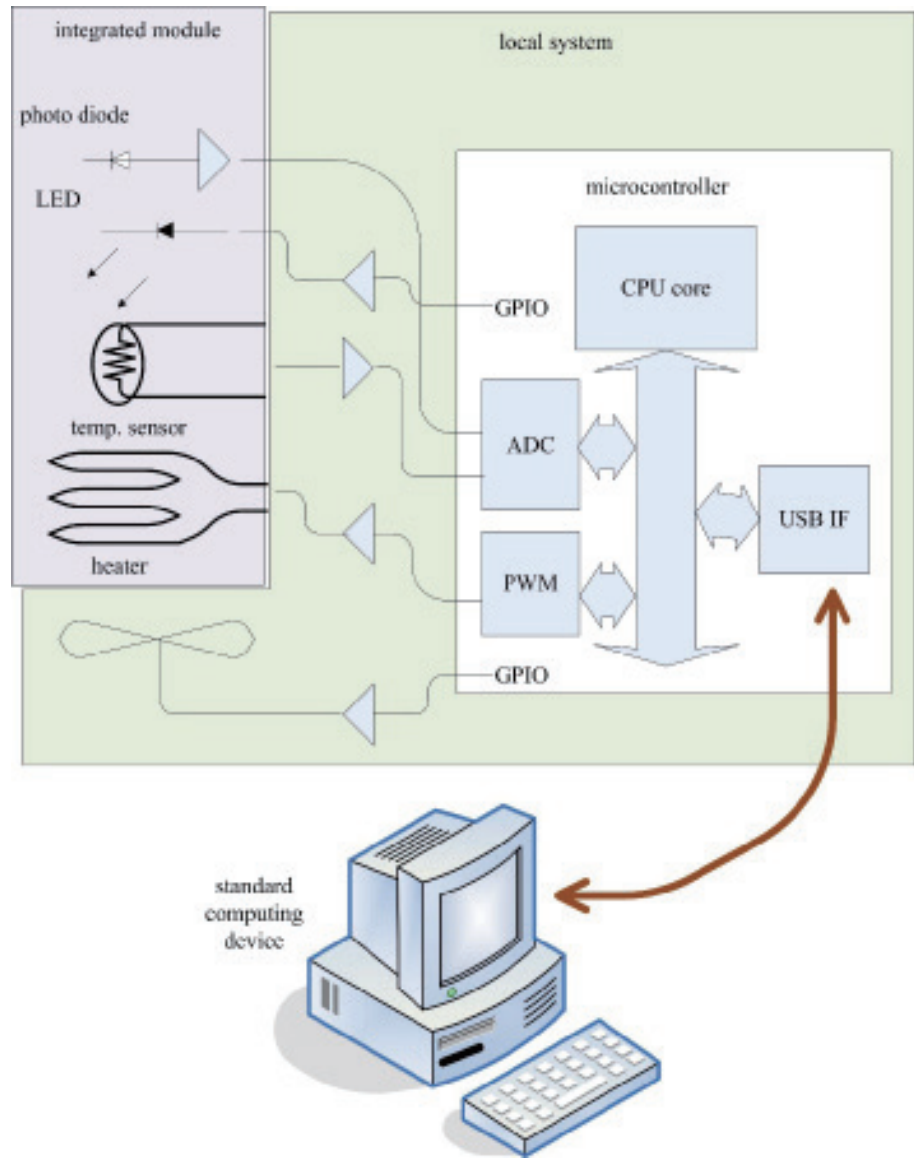

Fig. 7. (Color online) Block diagram of the realtime PCR system including the driving system of the integrated module.

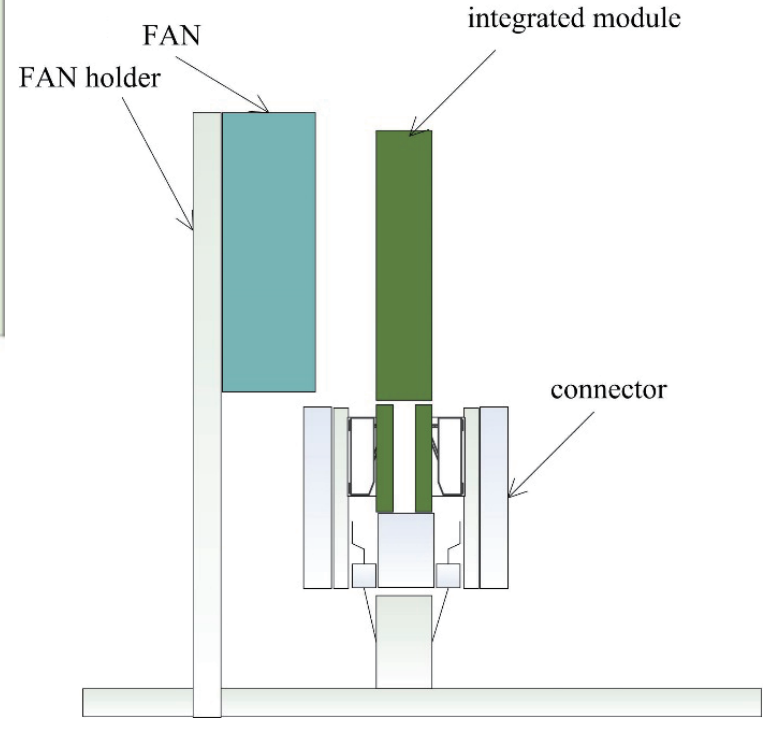

Fig. 8. (Color online) Arrangement of the fan and integrated module.

resistance of the thermistor, and the temperature was measured therefrom, and the temperature of the reagent was calculated through compensation as described in Sect. 2.1. Then, the calculated reagent temperature instead of the thermistor temperature was utilized for the proportional-integral-derivative (PID) control for driving the heater pattern or fan and sent back to the local system.

As shown in Fig. 9, the size of the system (overall size: $126 \times 60 \times 65 \mathrm{~mm}^{3}$ ) proposed in this paper is significantly smaller than that of the conventional real-time PCR system. Therefore, user space and portability are very high. In general, for real-time fluorescence measurements, the entire system must be shielded from light, like a darkroom. However, the proposed system does not require an external darkroom because it contains a housing that encloses the PCR chip and optical module.

\subsection{Air bubble problem}

A lot of heat was generated during PCR amplification. Because the optic module and PCR channel were close, air bubbles generated at $95{ }^{\circ} \mathrm{C}$ scattered the excitation and emission light 


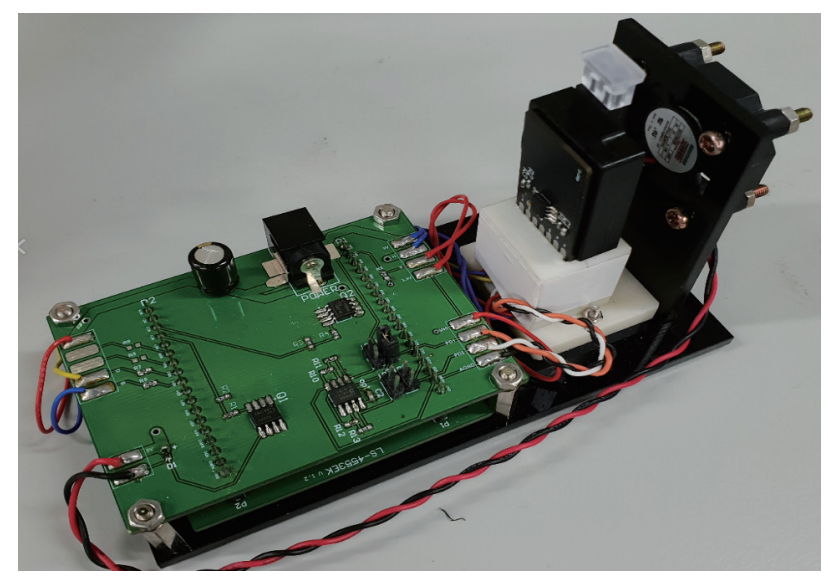

Fig. 9. (Color online) Implementation of the PCR chip driving system.

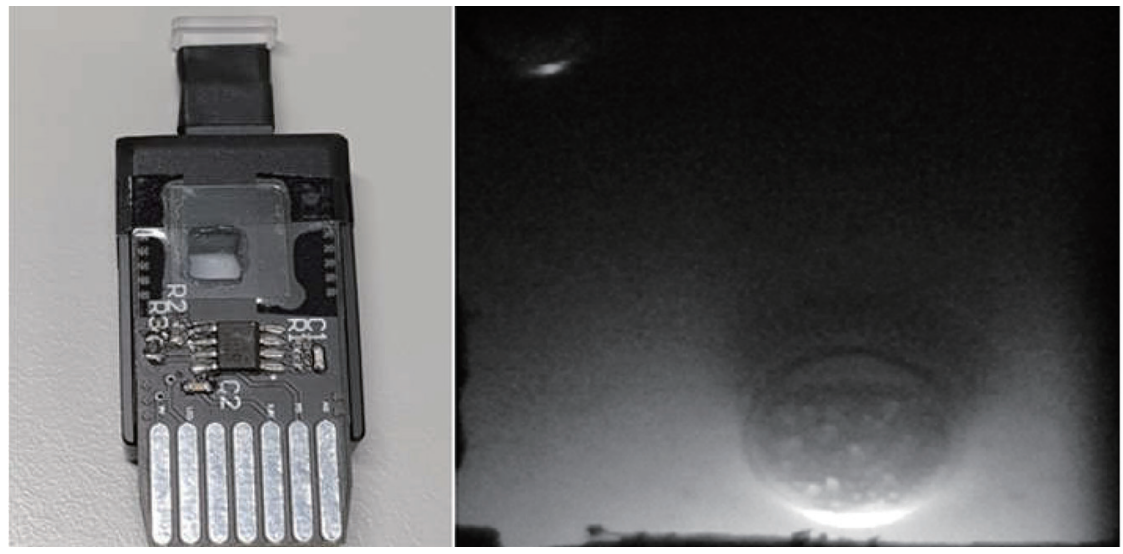

(a)

(b)

Fig. 10. (Color online) (a) Integrated module with an inspection hole and (b) the air bubble imaged through the hole.

and disturbed the fluorescence measurement. Figure 10(a) shows how to investigate the bubble behavior; the photodiode area of the optic PCB was cut out to reveal the square opening. Figure 10(b) shows air bubbles in the channel photographed through the opening shown in Fig. 10(a).

Since an air pocket was required to trap the air bubbles generated at the initial stage of the amplification, the shape of the chamber was that of a horn. That is, since the upper part of the integrated module had a horn-shaped air pocket, it was expected that the fluorescence detection performance could vary according to the LED positions. Figure 11 shows the integrated modules fabricated by placing the LEDs on the upper (a) and lower (b) parts. However, experiments according to the LED position showed almost the same fluorescence detection results, and the difference in the performance of amplification efficiency according to the position was not significant. Therefore, the configuration in Fig. 11(b) was employed for all the experiments. 


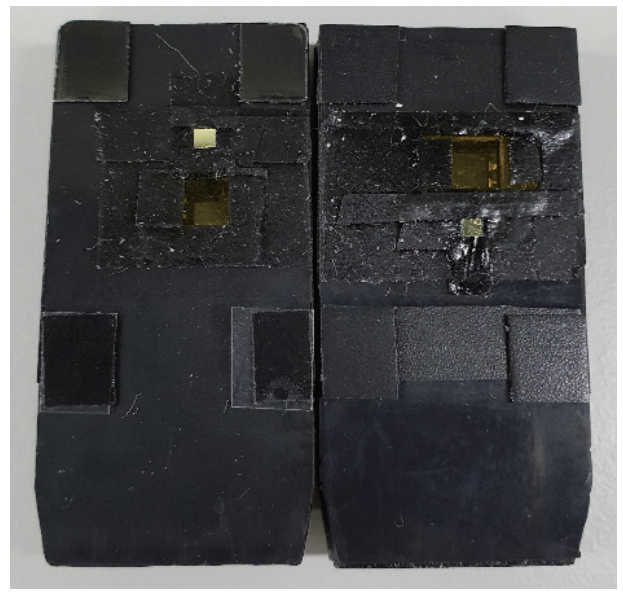

(a)

(b)

Fig. 11. (Color online) Photograph of actual integrated modules with LEDs and photodiodes in different positions.

\subsection{Amplification experiment}

Experiments were performed in a total volume of $36 \mu \mathrm{L}$ by adding $5.4 \mu \mathrm{L}$ of $0.1 \mathrm{ng} / \mu \mathrm{L}$ DNA (Chlamydia rachomatis), $18 \mu \mathrm{L}$ of a master mix, $3.6 \mu \mathrm{L}$ of DDW, and $9 \mu \mathrm{L}$ of a primer mix. In a primer mix that played a role in causing DNA synthesis, $10 \mu \mathrm{L}$ of primer $\mathrm{F}, 10 \mu \mathrm{L}$ of primer $\mathrm{R}, 4 \mu \mathrm{L}$ of fluorescent probe, and $376 \mu \mathrm{L}$ of DDW were added to prepare a total of $400 \mu \mathrm{L}$ (use $9 \mu \mathrm{L}$ each time). Table 1 shows an existing protocol with an air bubble problem, and Table 2 shows a modified protocol to solve this problem.

The PCR amplification protocol activates Uracil-DNA-Glycosylase (UDG) for $120 \mathrm{~s}$ at the first $50{ }^{\circ} \mathrm{C}$ and free denaturation at $95{ }^{\circ} \mathrm{C}$ for $600 \mathrm{~s}$. Then, denaturation was carried out at $95{ }^{\circ} \mathrm{C}$ for $15 \mathrm{~s}$. The final step extension was carried out at $60{ }^{\circ} \mathrm{C}$ for $60 \mathrm{~s}$. After the amplification experiment, the micro PCR amplification product was put in TBE $0.5 \mathrm{X}$ agarose gel and electrophoresed at $135 \mathrm{~V}$ for $25 \mathrm{~min}$. Commercial digital cameras (EOS 450D, Canon) were used for UV photography. To compare the brightness of all gels, the best setting for the gel condition was an aperture of 5.6, ISO 1600, and a focal length of $42 \mathrm{~mm}$.

\section{Result}

Figure 12 shows the relationship between the average thermocouple and thermistor temperatures for 15 chips. The relationship between the thermocouple and thermistor temperatures satisfied $y=0.985 x-0.09$ ( $y$ : thermocouple temperature, $x$ : thermistor temperature) and was very linear $\left(R^{2}=0.9996\right)$. Therefore, the temperature measured by the thermistor was multiplied by 0.985 to estimate the reagent temperature.

It was inferred that without compensation, the amplification efficiency was low, since the temperature tended to increase relatively later when no temperature compensation was applied. Therefore, temperature compensation was applied to all experiments.

As shown in Fig. 13, the overall nonsmoothness was due to the scattering of light by air bubbles. Not only that, but also because it seemed to escape the typical amplification curve, it 
Table 1

Existing PCR protocol.

\begin{tabular}{lcc}
\hline PCR process & $\begin{array}{c}\text { Target temperature } \\
\left({ }^{\circ} \mathrm{C}\right)\end{array}$ & Duration (s) \\
\hline Free denaturation & 50 & 180 \\
\hline \multirow{3}{*}{39 cycles } & 95 -denaturation & 10 \\
\cline { 2 - 3 } & 60-annealing & 30 \\
\cline { 2 - 3 } & 72 -extension & 30 \\
\hline
\end{tabular}

Table 2

Modified PCR protocol for air bubble problem.

\begin{tabular}{lcc}
\hline PCR process & $\begin{array}{c}\text { Target temperature } \\
\left({ }^{\circ} \mathrm{C}\right)\end{array}$ & Duration (s) \\
\hline UDG & 50 & 120 \\
\hline Free denaturation & 95 & 600 \\
\hline \multirow{2}{*}{39 cycles } & 95-denaturation & 15 \\
\cline { 2 - 3 } & 60-annealing, extension & 60 \\
\hline
\end{tabular}

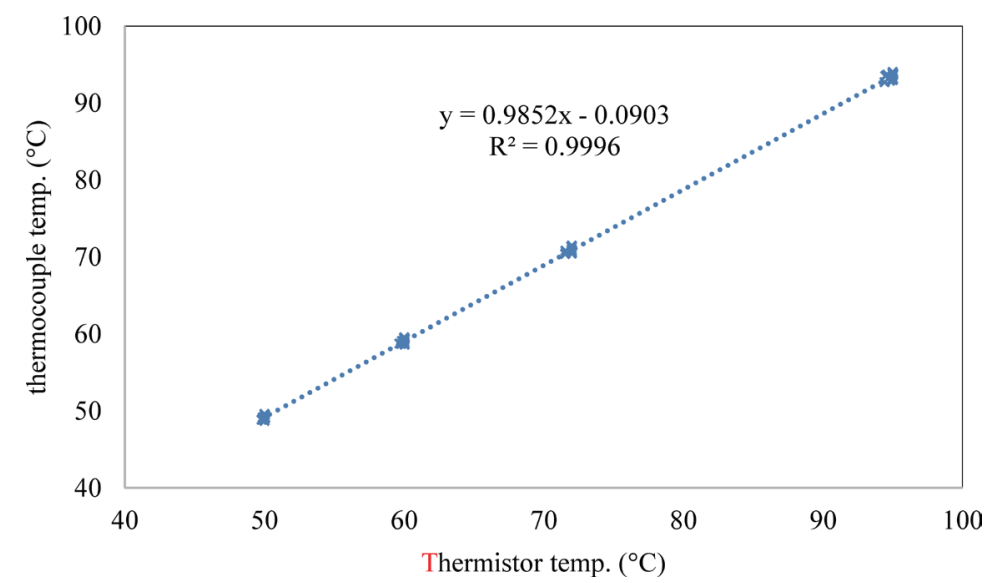

Fig. 12. (Color online) Relationship between thermistor and thermocouple temperatures.

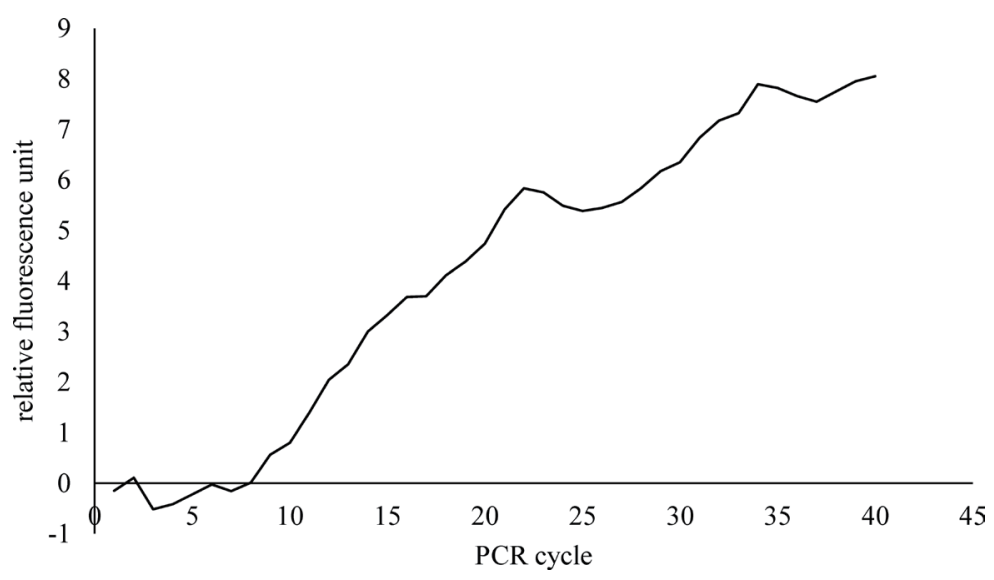

Fig. 13. Results of amplification due to scattering of light by air bubbles.

could be seen that the amplification did not work properly. Therefore, subsequent experiments used the modified protocol shown in Table 2.

Figure 14 shows the change in fluorescence brightness where both the modified protocol and the temperature compensation were applied and the LED was placed below the photodiode. The cycle where the brightness of the fluorescence rapidly changes was similar to that of the commercial real-time PCR, verifying that the proposed system performed well.

Figure 15 shows a gel image for comparing the band volumes of the DNA amplicons resulting from the commercial thermal cycler and proposed system. The band in the center 


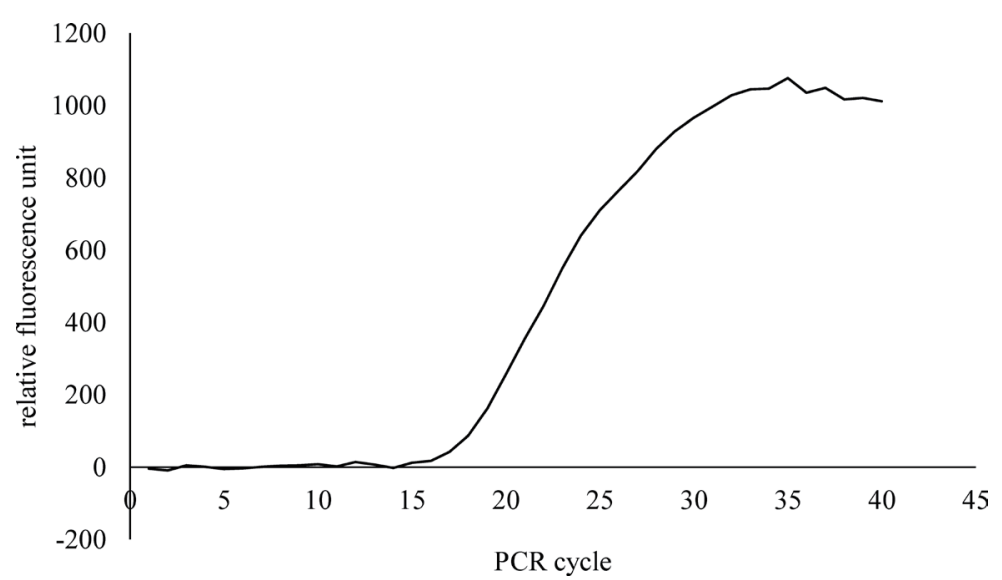

Fig. 14. PCR amplification result graph.

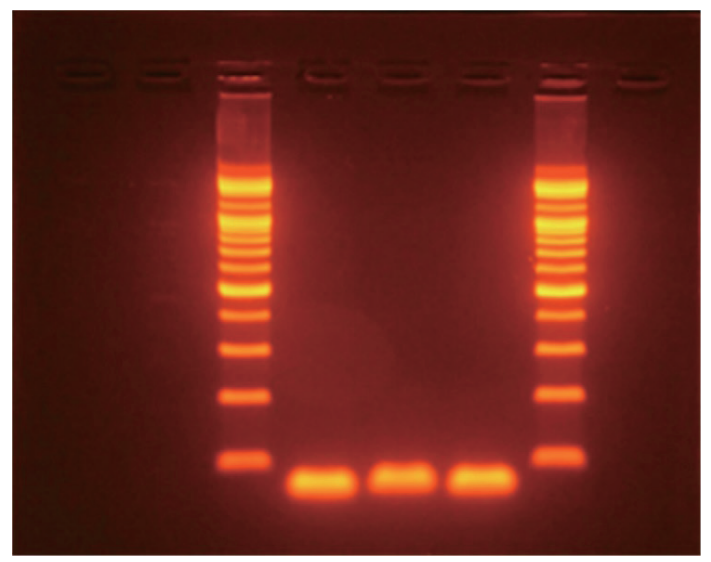

Fig. 15. (Color online) Gel image for comparison of the DNA amplicons from the proposed system and commercial thermal cycler. The center column is for the amplicon of the proposed system and its left and right columns are for those from the commercial thermal cycler.

column is for the amplicon from the proposed system and its left and right columns are for those from a commercial thermal cycler. The figure shows that the proposed system performs as well as the commercial thermal cycler. Figures 14 and 15 show that although the proposed system is very compact, it has compatible performance with conventional real-time PCR systems.

\section{Conclusions}

In this paper, we propose a compact real-time PCR system that can replace conventional real-time PCR systems, which are commonly large and expensive. The proposed real-time PCR system has no optical components such as lenses and mirrors that are usually inevitable for fluorescence measurements. An integrated module with a PCR chip and an optic module was fabricated to miniaturize the system. The temperature compensation was used to accurately estimate the temperature of the reactants and to increase the accuracy of the experiment. We also investigated the proper relative location of the LED and photodiode and developed a new 
protocol to minimize air bubbles during amplification. The proposed real-time PCR system highly miniaturized the optical parts of the conventional real-time PCR equipment.

\section{Acknowledgments}

This research was supported by the Government-wide R\&D Fund project for infectious disease research, HG18C0012, and by the Basic Science Research Program through the National Research Foundation of Korea (NRF) funded by the Ministry of Science, ICT \& Future Planning (NRF-2017R1A2B4002679).

\section{References}

1 C. Koo, M. Malapi-Wight, H. S. Kim, O. S. Cifci, V. L. Vaughn-Diaz, B. Ma, S. Kim, H. Abdel-Raziq, K. Ong, and Y.-K. Jo: PLoS One 8 (2013) e82704.

2 C. Zhang and D. Xing: Nucl. Acids Res. 35 (2007) 4223.

3 C. Zhang, D. Xing, and Y. Li: Biotechnol. Adv. 25 (2007) 483.

4 J. D. Kim, C. Y. Park, Y. S. Kim, M. S. Lee, and H. J. Song: Int. J. Eng. Technol. Innovation 8 (2018) 191.

5 E. Salm, Y.-S. Liu, D. Marchwiany, D. Morisette, Y. He, A. K. Bhunia, and R. Bashir: Biomed. Microdevices 13 (2011) 973.

6 J. Wu, R. Kodzius, K. Xiao, J. Qin, and W. Wen: Biomed. Microdevices 14 (2012) 179.

7 R. Kodzius, K. Xiao, J. Wu, X. Yi, X. Gong, I. G. Foulds, and W. Wen: Sens. Actuators, B 161 (2012) 349.

8 J.-S. Hwang, Y.-S. Kim, H.-J. Song, J.-D. Kim, and C.-Y. Park: Technol. Health Care 24 (2016) S139.

9 S. Cikos and J. Koppel: Anal. Biochem. 384 (2009) 1.

10 Q. Xiang, B. Xu, R. Fu, and D. Li: Biomed. Microdevices 7 (2005) 273.

11 Q. Xiang, B. Xu, and D. Li: Biomed. Microdevices 9 (2007) 443.

12 K. Sun, A. Yamaguchi, Y. Ishida, S. Matsuo, and H. Misawa: Sens. Actuators, B 84 (2002) 283.

13 D.-J. Lee, S.-Y. Kim, J.-D. Kim, Y.-S. Kim, H.-J. Song, and C.-Y. Park: Int. J. Bio-Sci. Bio-Technol. 5 (2013) 151.

14 T. Goldmann, A. Zyzik, S. Loeschke, W. Lindsay, and E. Vollmer: J. Biochem. Biophys. Methods 50 (2001) 91.

15 T. G. Porch and J. E. Erpelding: J. Biochem. Biophys. Methods 67 (2006) 1.

16 P. Neuzil, J. Pipper, and T. M. Hsieh: Mol. Biosyst. 2 (2006) 292.

17 C. D. Ahrberg, B. R. Ilic, A. Manz, and P. Neuzil: Lab Chip 16 (2016) 586.

18 J. Kim, D. Byun, M. G. Mauk, and H. H. Bau: Lab Chip 9 (2009) 606.

19 K. Shen, X. Chen, M. Guo, and J. Cheng: Sen. Actuators, B 105 (2005) 251.

20 D. Moschou, N. Vourdas, G. Kokkoris, G. Papadakis, J. Parthenios, S. Chatzandroulis, and A. Tserepi: Sen. Actuators, B 199 (2014) 470.

21 Y. S. Shin, K. Cho, S. H. Lim, S. Chung, S.-J. Park, C. Chung, D.-C. Han, and J. K. Chang: J. Micromech. Microeng. 13 (2003) 768.

22 M. Focke, D. Kosse, C. Müller, H. Reinecke, R. Zengerle, and F. von Stetten: Lab Chip 10 (2010) 1365.

23 A. C. Hatch, T. Ray, K. Lintecum, and C. Youngbull: Lab Chip 14 (2014) 562.

24 R. A. Mendoza-Gallegos, A. Rios, and J. L. Garcia-Cordero: Anal. Chem. 90 (2018) 5563.

\section{About the Authors}

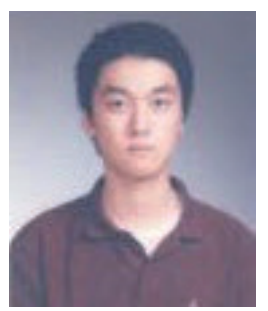

Deuk-Ju Lee received his M.S. degree from Hallym University. He is currently pursuing his Ph.D. degree in Convergence Software at Hallym University. He is a researcher in the Bio-IT Research Center at Hallym University. His research interests include embedded, biomedical, and optical systems. 


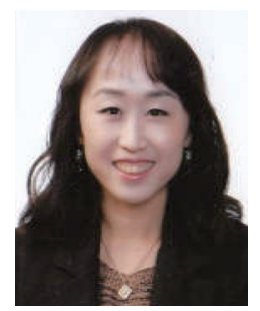

Ji-Soo Hwang received her M.S. and Ph.D. degrees in Computer Engineering from Hallym University. She is currently a research professor in the BioIT Research Center at Hallym University. Her recent research is focused on biomedical systems and bioinformatics.

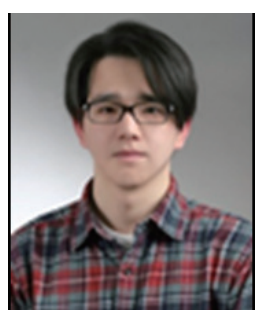

Ji-Seong Park is currently pursuing his Ph.D. degree in Convergence Software at Hallym University. His academic interests include embedded systems for biomedical applications.

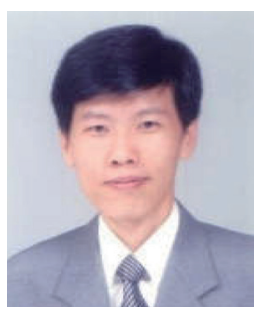

Chan-Young Park received his B.S. degree from Seoul National University and his M.S. and Ph.D. degrees from Korea Advanced Institute of Science and Technology in 1989 and 1995, respectively. From 1991 to 1999, he worked at Samsung Electronics. He is currently a professor in the School of Software at Hallym University. His research interests are in the areas of BioIT convergence, intelligent transportation systems, and sensors.

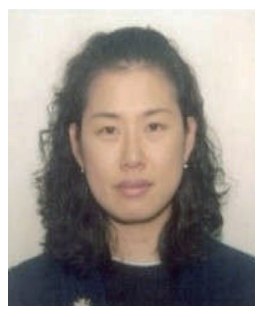

Hye-Jeong Song received her Ph.D. degree in Computer Engineering from Hallym University. She is currently a research professor in the Bio-IT Research Center at Hallym University. Her recent research is in the areas of biomedical systems and bioinformatics.

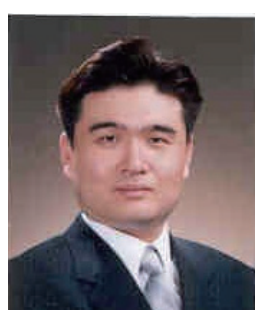

Yu-Seop Kim received his Ph.D. degree in Computer Engineering from Seoul National University. He is currenlty a professor in the School of Software at Hallym University. His research interests are in the areas of bioinformatics, computational intelligence, and natural language processing.

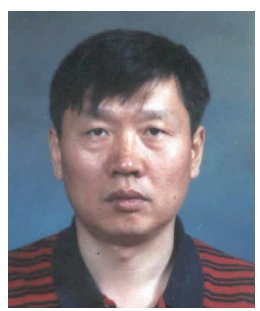

Jong-Dae Kim received his M.S. and Ph.D. degrees in Electrical and Electronics Engineering from Korea Advanced Institute of Science and Technology, Seoul, Korea, in 1984 and 1990, respectively. He worked for Samsung Electronics from 1988 to 2000 as an electronic engineer. He is currently a professor in the School of Software at Hallym University. His recent interests are focused on biomedical systems and bioinformatics. 